5. Acknowledgements. The author wishes to record his gratitude and sincere thanks to Mr. I. M. Khabaza formerly of the University of London Computer Unit ${ }^{3}$ for his constant interest during the period which he (the author) spent on the task of devising, refining, and running the search programmes, and for his very valuable help and advice on programming matters so freely given. The author wishes also to express his thanks to Professor R. A. Buckingham, Director of the Computer Unit, for allocating him sufficient machine time to enable him to complete the project quickly and thus be able to include the results in his Ph.D. thesis (University of London, 1963). Thanks are also due to Dr. M. Davies, Reader in Numerical Analysis at Battersea College, for providing free access to the College tapeediting equipment at all times and for his helpful preliminary advice.

Battersea College of Technology

London, S. W. 11

England

1. R. C. Bose, "On the application of the properties of Galois fields to the construction of hyper-Graeco-Latin squares," Sankhyā, v. 3, 1938, pp. 323-338.

2. R. C. Bose \& K. R. NAIR, "On complete sets of Latin squares," Sankhyā, v. 5, 1941, pp. 361-382. MR 4, 33.

3. A. D. KEEDWELL, "On the order of projective planes with characteristic," Rend. Mat. e Appl. Ser. V, v. 22, 1963, pp. 498-530.

4. W. LedermanN, Introduction to the Theory of Finite Groups, Oliver and Boyd, Edinburgh and Interscience, New York, 1949; 2nd ed., 1953. MR 10, 427; MR 14, 945.

5. H. B. MANN, "The construction of orthogonal Latin squares," Ann. Math Statist., v. 13, 1942, pp. 418-423. MR 4, 184; 340 .

${ }^{8}$ Now Reader in Computing Science at Queen Mary College, University of London.

\title{
Approximate Integration Formulas for Ellipses
}

\section{By Nancy Lee and A. H. Stroud}

1. Introduction. Here we give some approximate integration formulas of the form

$$
\begin{gathered}
I(f) \equiv \iint_{B_{B}} \frac{f(x, y)}{\sqrt{ }\left((x-c)^{2}+y^{2}\right) \sqrt{ }\left((x+c)^{2}+y^{2}\right)} d x d y \simeq \sum_{i=1}^{N} A_{i} f\left(x_{i}, y_{i}\right), \\
J(f) \equiv \int_{-\infty}^{\infty} \int_{-\infty}^{\infty} w(x, y) f(x, y) d x d y \simeq \sum_{i=1}^{N} A_{i} f\left(x_{i}, y_{i}\right), \\
w(x, y) \equiv \frac{D(x, y) \exp \left[-a D^{2}(x, y)\right]}{\sqrt{ }\left((x-c)^{2}+y^{2}\right) \sqrt{ }\left((x+c)^{2}+y^{2}\right)}, \\
D(x, y) \equiv \sqrt{ }\left((x-c)^{2}+y^{2}\right)+\sqrt{ }\left((x+c)^{2}+y^{2}\right) .
\end{gathered}
$$

Here $E_{B}$ is the interior of the ellipse with foci at $( \pm c, 0)$, semiminor axis $B$, and semimajor axis $\sqrt{ }\left(c^{2}+B^{2}\right)$. In $w(x, y), a$ is a positive constant. For both of these integrals we give integration formulas exact for all polynomials of degree $\leqq k$, $k=3,5,7$. These formulas are somewhat similar to formulas given by Hammer and Stroud [1] for a circle and square and were found by similar methods.

Received July 27, 1964. This work was supported in part by NSF grant GP-209. 
We have not encountered integrals of the form $I(f)$ and $J(f)$ in any practical problem but we believe that approximate integration formulas for these integrals will be useful since the weight functions in them become infinite at the points $( \pm c, 0)$. As a hypothetical example, the formulas we give here might be useful in problems in chemistry or physics which involve integrals of the form

$$
\iint_{\boldsymbol{E}_{\boldsymbol{B}}} G(x, y) d x d y
$$

where $G(x, y)$ is related to the repulsive force on a free particle $p$ due to two fixed particles located at $( \pm c, 0)$ under the assumption that the repulsive force on $p$ becomes infinite as $p$ approaches one of the fixed particles.

By transforming from rectangular to confocal elliptical coordinates, formulas for the integrals $I(f)$ and $J(f)$ can also be constructed by combinations of one-dimensional formulas. In this way one can obtain formulas of degree $2 h-1$ using $h^{2}$ points for $h=1,2,3, \cdots$. Formulas of this type for $I(f)$ have been discussed by Page [2] and will not be described here.

2. Description of the Formulas. We give two formulas for each of the degrees $3,5,7$ for each of the integrals $I(f)$ and $J(f)$. The formulas are given in terms of the monomial integrals $I_{j, k}$. Here $I_{j, k}$ denotes either $I\left(x^{j} y^{k}\right)$ or $J\left(x^{j} y^{k}\right), j, k=$ $0,1,2, \cdots$.

If at least one of the integers $j$ or $k$ is odd, then

$$
I\left(x^{j} y^{k}\right)=J\left(x^{j} y^{k}\right)=0 .
$$

The values of $I\left(x^{j} y^{k}\right), j$ and $k$ both even, are given by

$$
I\left(x^{2 n} y^{2 m}\right)=B\left(\frac{2 n+1}{2}, \frac{2 m+1}{2}\right) \sum_{k=0}^{n}\left(\begin{array}{l}
n \\
k
\end{array}\right) c^{2 n-2 k} g_{m+k},
$$

where

$$
\begin{aligned}
g_{n} & =A \sum_{k=0}^{n-1} \frac{(-1)^{2 k} c^{2 k} B^{2 n-2 k-1} P_{n, k}}{n(n-1) \cdots(n-k)}+\frac{(-1)^{n} c^{2 n} P_{n, n} L}{n !}, \\
A & =\sqrt{ }\left(c^{2}+B^{2}\right), \quad L=2 \log _{e}\left(\frac{A+B}{c}\right), \quad P_{n, k}=\left(\frac{2 n-1}{2}\right) \cdots\left(\frac{2 n-2 k+1}{2}\right) .
\end{aligned}
$$

Here $B(r, s)$ is the beta function $\Gamma(r) \Gamma(s) / \Gamma(r+s)$.

Thus $I(1)=\pi L$.

The values of $J\left(x^{j} y^{k}\right)$ for $j$ and $k$ both even are

$$
\begin{aligned}
& J\left(x^{2 n} y^{2 m}\right) \\
& =a^{-.5} e^{-4 a c} B\left(\frac{2 n+1}{2}, \frac{2 m+1}{2}\right) \sum_{k=0}^{n}\left(\begin{array}{l}
n \\
k
\end{array}\right) c^{2 n-2 k}(4 a)^{-m-k} \Gamma\left(\frac{2 m+2 k+1}{2}\right) .
\end{aligned}
$$

In Table 1 we give numerical values of the constants in the formulas for $I(f)$ for $B=1, c=1$, and in Table 2 numerical values for $J(f)$ for $c=1, a=1 / 4$. 
TABLe 1

Formulas for $I(f), B=1, c=1$

Formula 3a

$$
\begin{aligned}
& u=1.141174027799650 \quad v=0.549798291853001 \\
& A_{1}=1.384458393024340 \\
& A_{1}=1.384458393024340 \\
& v=0.388766100454037 \\
& A_{1}=1.191157269603166 \\
& \eta=0.657867463793369 \\
& A_{2}=0.483481509383302
\end{aligned}
$$

$v=0.803909065610874$

$\lambda=1.012197157907448$

$A_{0}=1.221592995357823$

$u_{1}=1.246009745849288$

$u_{2}=0.780689798095836$

$v_{1}=0.895112350759653$

$v_{2}=0.394559771541860$

$\lambda=0.900546669274181$

$$
A_{5}=
$$

Formula 5b

$$
\begin{aligned}
A_{1} & =0.398360298916025 \\
\eta & =0.302513408229517 \\
A_{2} & =0.879879994726872
\end{aligned}
$$

Formula 7a

$$
\begin{aligned}
A_{1} & =0.438093548819901 \\
A_{2} & =0.971706127419465 \\
A_{3} & =0.163122086390356 \\
A_{4} & =0.541777751729327 \\
\eta & =0.557659666410276
\end{aligned}
$$

Formula 7b

$$
\begin{aligned}
u_{1} & =1.271634501705140 \\
u_{2} & =0.821020360201681 \\
v_{1} & =0.910429182160393 \\
v_{2} & =0.440808525551630 \\
\lambda & =0.900546669274181 \\
A_{0} & =0.211469951435905
\end{aligned}
$$

$A_{1}=0.366749953216258$

$A_{2}=1.007804731117124$

$A_{3}=0.144612593026746$

$A_{4}=0.489797261280969$

$\eta=0.557659666410276$

$A_{5}=0.327108635844816$

The formulas are:

Formula $3 \mathrm{a}, 4$ points, degree 3 :

$$
\begin{array}{cc}
\text { Point } & \text { Coefficient } \\
( \pm u, 0) & A_{1} \\
(0, \pm v) & A_{1} \\
u^{2}=\frac{2 I_{20}}{I_{00}}, \quad v^{2}=\frac{2 I_{02}}{I_{00}}, & A_{1}=\frac{I_{00}}{4} ;
\end{array}
$$

Formula $3 b, 4$ points, degree 3 :

$$
\begin{array}{cl}
( \pm u, \pm v) & A_{1} \\
u^{2}=\frac{I_{20}}{I_{00}}, \quad v^{2}=\frac{I_{02}}{I_{00}}, \quad A_{1}=\frac{I_{00}}{4} ;
\end{array}
$$


TABLE 2

Formulas for $J(f), c=1, a=0.25$

Formula 3a

$$
\begin{aligned}
& u=1.224744871391589 \quad v=0.707106781186548 \\
& A_{1}=1.024236695866873 \\
& \text { Formula } 3 b \\
& u=0.866025403784439 \quad v=0.500000000000000 \\
& A_{1}=1.024236695866873 \\
& \text { Formula 5a } \\
& u=1.243163121016122 \\
& \lambda=0.790569415042095 \\
& A_{0}=1.566479652502276 \\
& v=1.374368541872554 \\
& \lambda=1.172603939955857 \\
& A_{0}=1.566479652502276 \\
& u_{1}=1.917739116886260 \\
& u_{2}=0.934449448785687 \\
& v_{1}=1.854770545973768 \\
& v_{2}=0.617009547822385 \\
& \lambda=1.244989959798873 \\
& A_{5}=0.098333016116251 \\
& A_{1}=0.810017256208442 \\
& \eta=1.060660171779821 \\
& A_{2}=0.227608154637083 \\
& A_{1}=0.147884442718746 \\
& \eta=0.456435464587638 \\
& A_{2}=0.558674561381931 \\
& u_{1}=1.975911856128909 \\
& u_{2}=0.955805485502959 \\
& v_{1}=1.901481972888572 \\
& v_{2}=0.661716141789722 \\
& \lambda=1.244989959798873 \\
& A_{0}=0.185180735950124 \\
& A_{1}=0.044100110335543 \\
& A_{2}=1.159574673340265 \\
& A_{3}=0.019625337242702 \\
& A_{4}=0.535916870607671 \\
& \eta=1.024695076595960 \\
& A_{5}=0.098333016116251
\end{aligned}
$$

Formula 5a, 7 points, degree 5 :

$$
\begin{aligned}
& (0,0) \\
& A_{0} \\
& ( \pm u, 0) \\
& \begin{array}{c}
( \pm \lambda, \pm \eta) \quad A_{2} \\
u^{2}=\frac{I_{40} I_{04}-I_{22}^{2}}{I_{20} I_{04}-I_{02} I_{22}}, \quad \lambda^{2}=\frac{I_{22}}{I_{02}}, \quad \eta^{2}=\frac{I_{04}}{I_{02}},
\end{array} \\
& A_{1} \\
& A_{1}=\frac{\left(I_{20} I_{04}-I_{02} I_{22}\right)^{2}}{2 I_{04}\left(I_{40} I_{04}-I_{22}^{2}\right)}, \quad A_{2}=\frac{I_{02}^{2}}{4 I_{04}}, \quad A_{0}=I_{00}-2 A_{1}-4 A_{2} \text {; } \\
& (0,0) \quad A_{0} \\
& (0, \pm v) \quad A_{1} \\
& ( \pm \lambda, \pm \eta) \quad A_{2} \\
& v^{2}=\frac{I_{40} I_{04}-I_{22}^{2}}{I_{40} I_{02}-I_{20} I_{22}}, \quad \lambda^{2}=\frac{I_{40}}{I_{20}}, \quad \eta^{2}=\frac{I_{22}}{I_{20}}, \\
& A_{1}=\frac{\left(I_{40} I_{02}-I_{20} I_{22}\right)^{2}}{2 I_{40}\left(I_{40} I_{04}-I_{22}^{2}\right)} \cdot \quad A_{2}=\frac{I_{20}^{2}}{4 I_{40}} \quad A_{0}=I_{00}-2 A_{1}-4 A_{2}
\end{aligned}
$$


Formula 7a, 12 points, degree 7:

$\begin{array}{cc}\left( \pm u_{1}, 0\right) & A_{1} \\ \left( \pm u_{2}, 0\right) & A_{2} \\ \left(0, \pm v_{1}\right) & A_{3} \\ \left(0, \pm v_{2}\right) & A_{4} \\ ( \pm \lambda, \pm \eta) & A_{5} \\ \lambda^{2}=\frac{I_{42}}{I_{22}}, \quad \eta^{2}=\frac{I_{24}}{I_{22}}, & A_{5}=\frac{I_{22}^{3}}{4 I_{42} I_{24}} .\end{array}$

$u_{1}^{2}, u_{2}^{2}$ are roots of $u^{4}+c_{1} u^{2}+c_{0}=0$, where

$$
\begin{gathered}
c_{0}=\left[\left[I_{20}-4 A_{5} \lambda^{2}\right]\left[I_{60}-4 A_{5} \lambda^{6}\right]-\left[I_{40}-4 A_{5} \lambda^{4}\right]^{2}\right] / D_{1}, \\
c_{1}=\left[\left[I_{20}-4 A_{5} \lambda^{2}\right]\left[I_{40}-4 A_{5} \lambda^{4}\right]-k_{1}\left[I_{60}-4 A_{6} \lambda^{6}\right]\right] / D_{1}, \\
D_{1}=k_{1}\left[I_{40}-4 A_{5} \lambda^{4}\right]-\left[I_{20}-4 A_{5} \lambda^{2}\right]^{2}, \\
A_{1}=\left[I_{20}-4 A_{5} \lambda^{2}-k_{1} u_{2}^{2}\right] /\left[2\left(u_{1}^{2}-u_{2}^{2}\right)\right], \\
A_{2}=\left[I_{20}-4 A_{5} \lambda^{2}-k_{1} u_{1}^{2}\right] /\left[2\left(u_{2}^{2}-u_{1}^{2}\right)\right], \\
k_{1}=(2 / 3)\left[I_{00}-4 A_{5}\right] .
\end{gathered}
$$

$v_{1}^{2}, v_{2}^{2}$ are roots of $v^{4}+d_{1} v^{2}+d_{0}=0$, where

$$
\begin{gathered}
d_{1}=\left[\left[I_{02}-4 A_{5} \eta^{2}\right]\left[I_{06}-4 A_{5} \eta^{6}\right]-\left[I_{04}-4 A_{5} \lambda^{4}\right]^{2}\right] / D_{2}, \\
d_{0}=\left[\left[I_{02}-4 A_{5} \eta^{2}\right]\left[I_{04}-4 A_{5} \eta^{4}\right]-k_{2}\left[I_{06}-4 A_{5} \eta^{6}\right]\right] / D_{2}, \\
D_{2}=k_{2}\left[I_{04}-4 A_{5} \eta^{4}\right]-\left[I_{02}-4 A_{5} \eta^{2}\right]^{2}, \\
A_{3}=\left[I_{02}-4 A_{5} \eta^{2}-k_{2} v_{2}^{2}\right] /\left[2\left(v_{1}^{2}-v_{2}^{2}\right)\right], \\
A_{4}=\left[I_{02}-4 A_{5} \eta^{2}-k_{2} v_{1}^{2}\right] /\left[2\left(v_{2}^{2}-v_{1}^{2}\right)\right], \\
k_{2}=(1 / 3)\left[I_{00}-4 A_{5}\right] .
\end{gathered}
$$

Formula $7 \mathrm{~b}, 13$ points, degree 7 :

$$
\begin{array}{cc}
(0,0) & A_{0} \\
\left( \pm u_{1}, 0\right) & A_{1} \\
\left( \pm u_{2}, 0\right) & A_{2} \\
\left(0, \pm v_{1}\right) & A_{3} \\
\left(0, \pm v_{2}\right) & A_{4} \\
( \pm \lambda, \pm \eta) & A_{5}
\end{array}
$$

The parameters in this formula are determined by the same equations as the parameters in Formula 7a except we use

$$
\begin{gathered}
k_{1}=0.65\left[I_{00}-4 A_{5}\right], \quad k_{2}=0.30\left[I_{00}-4 A_{5}\right], \\
A_{0}=I_{00}-2\left(A_{1}+A_{2}+A_{3}+A_{4}\right)-4 A_{5} .
\end{gathered}
$$


3. Concluding Remarks. We can obtain formulas similar to those given here for any region (and weight function) which has the same symmetries as the ellipse. We need only substitute the appropriate monomial integrals $I_{2 n, 2 m}$ in the expressions given.

It should also be noted that the formulas of degree 7 are not unique. Similar formulas can be obtained by choosing different values for the quantities $k_{1}$ and $k_{2}$. Various 12-point formulas are obtained by choosing $k_{1}$ and $k_{2}$ to satisfy

$$
k_{1}+k_{2}=I_{00}-4 A_{5} .
$$

Although there is this free parameter in the 12-point formulas we believe it is not possible to obtain a formula of degree 7 using fewer points.

Computation Center

University of Kansas

Lawrence, Kansas

1. P. C. Hammer \& A. H. Stroud, "Numerical evaluation of multiple integrals. II," $M T A C$, v. 12,1958 , pp. $272-280$. MR 21 *970.

2. L. G. PAGE, "Numerical integration over ellipses," Master's Thesis, Univ. of Kansas, Lawrence, Kan., 1964.

\section{Improved Asymptotic Expansion for the Exponential Integral with Positive Argument}

\section{By Donald van Zelm Wadsworth}

The usual asymptotic approximation to the exponential integral can be markedly improved, for the case with positive real argument, by adding a simple correction term as shown below. Similar results for the error function with imaginary argument (essentially the same as Dawson's function) are given in [1].*

By definition, the exponential integral with positive real argument is

$$
\operatorname{Ei}(x)=-\int_{-x}^{\infty} t^{-1} e^{-t} d t=-\int_{L} t^{-1} e^{-t} d t-i \pi
$$

The line integral along the real axis from $-x$ to $\infty$ is a Cauchy principal value since there is a pole at the origin. The path of integration $L$ goes from $-x$ to $\infty$, passing above the origin. Repeated partial integration of the infinite integral yields $\mathrm{Ei}(x)=$ $E_{n}(x)+e_{n}(x)$, where

$$
E_{n}(x)=x^{-1} e^{x} \sum_{0}^{n-1} m ! x^{-m}
$$

is the asymptotic approximation for the interval $\left(n-\frac{1}{2}\right) \leqq x<\left(n+\frac{1}{2}\right)$, and

$$
e_{n}(x)=-(-)^{n} n ! \int_{L} t^{-n-1} e^{-t} d t-i \pi
$$

Received November 2, 1964.

${ }^{*}$ The correction term derived in [1] could also be obtained, in a less direct fashion, from the Chebyshev polynomial expansions for Dawson's function given in [2]. 